\title{
Perfil dos estudantes de graduação em Odontologia: motivações e expectativas da profissão
}

\author{
Gélica Lima Granja*; Jhonatan Thiago Lacerda Santos*; Rosálya Coura Mariz**; Ângela Toshie \\ Araki***; Stheshy Vieira e Souza****; Jocianelle Maria Felix Fernandes Nunes*****; Fátima Roneiva \\ Alves Fonseca******
}

\author{
* Estudante de Odontologia pelas Faculdades Integradas de Patos \\ ** Professora do Curso de Odontologia das Faculdades Integradas \\ de Patos \\ *** Professora adjunta da Universidade Cruzeiro do Sul \\ **** Cirurgiã-dentista \\ ****** Professora Adjunta, Departamento de Clínica e Odontologia \\ Social da Universidade Federal da Paraíba \\ Professora do Curso de Odontologia da UFCG
}

Recebido em 09/09/2016. Aprovado em 02/12/2016.

\begin{abstract}
RESUMO
O objetivo deste estudo foi registrar o perfil dos estudantes de graduação em Odontologia quanto às motivações e expectativas. Foram analisados 98 alunos do curso de Odontologia das Faculdades Integradas de Patos, que preencheram um questionário com perguntas objetivas e subjetivas. Os resultados revelam que $68,4 \%$ eram do sexo feminino e a maioria do Estado da Paraíba $(67,3 \%$ ). Cerca de $80 \%$ fizeram vestibular em outra instituição e $40,8 \%$ fizeram de dois a três processos seletivos. A renda familiar predominante foi de 3 a 6 salários mínimos (40,8\%). A expectativa dos participantes foi de formação profissional voltada para o trabalho $(70,4 \%)$, o motivo de escolha principal foi a realização pessoal e profissional $(69,4 \%)$, sendo a Odontologia uma dentre outras carreiras que poderia satisfazê-los $(67,3 \%)$. Os resultados revelam que boa parte dos entrevistados estão motivados na área profissional e pessoal e suas expectativas são as melhores voltadas para a formação acadêmica e o trabalho. Há predominância de mulheres, a maioria dos estudantes acredita que o mercado de trabalho está saturado, mas almeja trabalhar seja em consultório individual, particular, público ou convênios. A vocação foi a maior explicação para a procura do curso, e a necessidade de continuar estudando é preocupação constante.
\end{abstract}

Descritores: Estudantes de Odontologia. Formação de Recursos Humanos. Mercado de Trabalho.

\section{INTRODUÇÃO}

A Odontologia encontra-se em mudança no Brasil, começando no setor de novas tecnologias, que são lançadas no mercado a cada mês, quanto no rumo que o profissional deve tomar diante das dificuldades do mercado de trabalho. Desta forma, existe uma conjuntura que reúne desafios de várias ordens.

O mercado de trabalho tornou-se extremamente competitivo, criando um processo amplo de busca e experimentação de diferentes mecanismos de concorrência que se expressam pela 
sensibilização geral às demandas por novas habilidades.

Evidencia-se que os profissionais necessitam incorporar, em seus processos de trabalho, posturas relacionais sustentadas pela ética e comunicação, com referenciais científicos, interpessoais (afetivo/psicológico), pedagógicos, antropológicos e sociológicos ${ }^{1}$.

Os novos profissionais devem se preparar e conhecer as dificuldades e vicissitudes que poderão advir para, desta forma, encontrar meios de transpô-las, passando a viver uma fase da profissão que, no futuro, será chamada a "Era da Superação em Odontologia"2.

A educação é um meio básico para se conseguir ascensão social e intelectual. Para que um indivíduo possa aprender, é preciso um controle sobre sua mente, proporcionando abertura para novas concepções. Com o controle físico e emocional, haverá possibilidades de mudança no seu cotidiano.

Para o ensino superior, sabe-se que a aprendizagem em sala de aula enfatiza informações técnicas e treina especificamente para o exercício da profissão. O papel da universidade é formar um profissional com perfil bem definido, buscando um ensino críticoreflexivo na área e contribuindo para uma inserção dos profissionais no mercado de trabalho regida pela ética da cidadania coletiva ${ }^{3}$.

Com relação à instituição formadora, esta deve estar aberta às demandas sociais, capaz de produzir conhecimento relevante e útil. Como consequência, deve ser priorizada a atenção à saúde universal e com qualidade, com ênfase na promoção da saúde e prevenção das doenças ${ }^{4}$.

Com todas as dificuldades que vêm ocorrendo no campo de trabalho odontológico na esfera privada, as quais têm provocado certo nível de insatisfação da categoria, não se pode negar que o momento é de mudanças, que se configuram tanto na forma de se lidar com as instabilidades da atuação em nível privado, quanto na busca de novos caminhos para atuação profissional. Nessa perspectiva, a esfera pública tem propiciado importantes espaços de atuação ${ }^{1}$.

Esse estudo visa fornecer informações acerca do perfil dos estudantes de Odontologia das Faculdades Integradas de Patos, suas motivações e expectativas, destacando questões acerca da preparação adequada dos alunos para o mercado de trabalho.

\section{MATERIAL E MÉTODOS}

Este é um estudo descritivo do tipo transversal, realizado em 2013, com estudantes do $1^{\circ}$ ao $8^{\circ}$ período do Curso de Odontologia das Faculdades Integradas de Patos/PB.

Foram incluídos na pesquisa alunos regularmente matriculados no turno da manhã e que aceitaram participar por meio da assinatura do Termo de Consentimento Livre e Esclarecido (TCLE). Os alunos menores de 18 anos foram excluídos da pesquisa.

Um questionário preconizado por $\operatorname{Machado}^{5}$ (2010) foi aplicado, o qual abordou os seguintes eixos: dados relativos à classificação econômica das famílias dos estudantes; acesso a bens de consumo e transporte; exercício de trabalho remunerado extracurricular; motivo de escolha profissional; expectativas do mercado de trabalho em Odontologia e intenção de estudos complementares de pós-graduação.

Após a coleta de dados os questionários foram digitados e o cálculo erro de digitação foi realizado com $10 \%$ das fichas, escolhidas de forma aleatória, devendo situar-se abaixo de $1 \%$. Os dados foram trabalhados pela estatística descritiva, por meio da distribuição absoluta e percentual, utilizando o pacote estatístico Statistical Program for Social Science (SPSS) versão 20.0 .

Para a execução do estudo foram obedecidos todos os critérios prescritos pela 
Resolução 196/96, do Conselho Nacional de Saúde (CNS), a qual versa sobre a ética em pesquisa com seres humanos. Esta pesquisa foi aprovada Comitê de Ética e Pesquisa envolvendo seres humanos das Faculdades Integradas de Patos, parecer 191/2012.

\section{RESULTADOS}

Dentre os 98 alunos entrevistados, a maioria foi do sexo feminino $(68,4 \%)$, do estado da Paraíba $(67,3 \%)$ e, destes, $96,9 \%$ era do interior do Estado. A maioria dos entrevistados estudou em escolas privadas $(50,0 \%)$, cerca de $80 \%$ fizeram vestibular em outra instituição e $40,8 \%$ participaram de dois a três processos seletivos.

A renda familiar predominante foi de três a seis salários mínimos (40,8\%). O grau de escolaridade materna que se destacou foi o superior completo $(39,8 \%)$. Em contrapartida, a escolaridade paterna predominante foi ensino médio incompleto $(23,5 \%)$.

Observou-se que $86,7 \%$ dos alunos de graduação de Odontologia não possuem nenhum tipo de trabalho remunerado (tabela 1).

A expectativa quanto à formação profissional, foi, em sua maioria, voltada para o trabalho $(70,4 \%)$, o principal motivo de escolha foi a realização pessoal e profissional $(69,4 \%)$, sendo a Odontologia uma dentre outras carreiras que poderia satisfazê-los $(67,3 \%)$ (tabela 2). Observou-se que a maioria dos estudantes de odontologia escolheu o curso por vocação $(44,9 \%)$, sendo a principal finalidade prevenir e manter a saúde bucal $(64,3 \%)$ (tabela 3$)$.

A maioria dos estudantes pretende, após o término do curso, buscar cursos de pósgraduação $(69,4 \%)$. As especialidades mais citadas foram Ortodontia $(9,2 \%)$ e Cirurgia e Traumatologia Buco-Maxilo-Faciais (16,3\%); $26,5 \%$ ainda não escolheram e pretendem fazer cursos em outras especialidades.
A principal dificuldade que os alunos imaginam enfrentar no exercício profissional é a saturação do mercado de trabalho (46,9\%). A maioria relatou desejo de trabalhar em consultório particular e no serviço público (81,6\%). Após 5 anos de formado o desejo dos entrevistados é receber remuneração acima de $\mathrm{R} \$$ $5.000,00(51,0 \%)$.

\section{DISCUSSÃO}

De acordo com a estratificação da amostra pelo gênero, observou-se uma maior prevalência do gênero feminino, visto que as mulheres são maioria na população isso se reflete nas faculdades de Odontologia ${ }^{3,6-9}$.

$O$ perfil dos entrevistados é de um estudante do mesmo estado em que se situa a faculdade, que cursou o segundo grau em escola privada, que não possui trabalho remunerado, cuja mãe e pai possuem formação superior e nível médio, respectivamente, com renda familiar média de 3 a 6 salários mínimos, o que está de acordo com estudos prévios ${ }^{3,6-8,10}$.

Quando questionados sobre qual a principal finalidade da Odontologia, a maioria dos entrevistados destacou prevenção e manutenção de saúde bucal. Em um estudo no Estado de Santa Catarina, estas finalidades foram apontadas como principais por menor parcela dos estudantes $(28,2 \%)^{11}$.

No presente estudo identificou-se que o motivo da escolha pelo curso de Odontologia foi por vocação $(44,9 \%)$, o que está de acordo com a literatura $3,6,7,9,10,12,13$. Outros estudos relatam também admiração ${ }^{7}$ e afinidade $^{14}$.

A maior expectativa em relação ao curso de Odontologia foi de formação profissional voltada para o trabalho, com isso a Odontologia significou uma das dentre outras carreiras que poderia satisfazê-los. Outros estudos mostram que $64 \%$ dos graduandos escolheram a Odontologia como primeira opção de carreira ${ }^{14,15}$ 
Tabela 1. Distribuição absoluta e percentual da renda familiar, escolaridade materna, escolaridade paterna e trabalho remunerado dos participantes.

\begin{tabular}{lcc}
\hline & $\mathbf{n}$ & $\mathbf{\%}$ \\
\hline Renda & 1 & 1,0 \\
1 salario & 31 & 31,6 \\
1 a 3 salario & 40 & 40,8 \\
3 a 6 salario & 26 & 26,5 \\
Acima de 6 & $\mathbf{9 8}$ & $\mathbf{1 0 0}$ \\
Total & & \\
\hline Escolaridade Materna & 6 & 6,1 \\
Fundamental incompleta & 9 & 9,2 \\
Fundamental completo & 11 & 11,2 \\
Médio incompleta & 26 & 26,5 \\
Médio completo & 7 & 7,1 \\
Superior incompleto & 39 & 39,8 \\
Superior completo & $\mathbf{9 8}$ & $\mathbf{1 0 0}$ \\
Total & & \\
\hline Escolaridade Paterna & 2 & 2,0 \\
Analfabeto & 15 & 15,3 \\
Fundamental incompleto & 8 & 8,2 \\
Fundamental completo & 11 & 11,2 \\
Médio incompleto & 23 & 23,5 \\
Médio completo & 11 & 11,2 \\
Superior incompleto & 20 & 20,4 \\
Superior completo & 8 & 8,2 \\
Não informa & $\mathbf{9 8}$ & $\mathbf{1 0 0}$ \\
Total & & \\
Trabalho remunerado & 85 & 86,7 \\
Não & 5 & 5,1 \\
Sim parcial & 3 & 3,1 \\
Sim total & 5 & 5,1 \\
Sim, eventualmente & 98 & 100 \\
Total & & \\
\hline & & \\
\hline & & \\
\hline & & \\
\hline
\end{tabular}

Dos 98 estudantes que participaram da pesquisa, $69,4 \%$ pretendem fazer aperfeiçoamento e especialização ${ }^{6,12}$. As três principais áreas citadas pelos alunos entrevistados são Cirurgia e Traumatologia Buco-Maxilo-Faciais, Ortodontia, Dentística, Odontopediatria e Endodontia. Uma parcela dos estudantes $(26,5 \%)$ ainda não decidiu quanto à especialidade. Estes resultados se assemelham aos de outros estudos, nos quais a maioria dos estudantes pretendem fazer pós-graduação e as três principais áreas de preferência entre os alunos entrevistados também foram Cirurgia e Traumatologia Buco-Maxilo-
Faciais, Ortodontia e Dentística ${ }^{3,16}$. Outro estudo realizado na Bahia corrobora com o resultado desta pesquisa, observando que a maioria dos estudantes pretendem se especializar em Ortodontia $(12,5 \%)$ e Cirurgia $(10 \%)^{6}$.

Quanto à pretensão salarial após cinco anos de formados, a metade afirmou querer ganhar mais que $\mathrm{R} \$ 5,000,00$. Ao associar este dado com outros estudos pode-se observar que, após dez anos de profissão, o rendimento médio mensal é de R\$ 5.000.00, portanto a expectativa dos entrevistados, diante da questão financeira não se distância da realidade encontrada na literatura ${ }^{6,17}$. 
Tabela 2. Expectativas, motivos da escolha e representação do curso.

\begin{tabular}{lcc}
\hline & $\mathbf{n}$ & $\mathbf{\%}$ \\
\hline Expectativa & & \\
Formação profissional para trabalho & 69 & 70,4 \\
Aquisição ampla & 1 & 1,0 \\
Atividade pratica atual & 5 & 5,1 \\
Grau de instrução & 5 & 5,1 \\
Compreender melhor o mundo & 1 & 1,0 \\
Melhor salário & 12 & 12,2 \\
Outro & 5 & 5,1 \\
Total & $\mathbf{9 8}$ & $\mathbf{1 0 0}$ \\
\hline Motivo de escolha & & \\
Realização pessoal/profissional & 68 & 69,4 \\
Conforto financeiro & 14 & 14,3 \\
Influência amigos/parentes & 5 & 5,1 \\
Interesse atuar comunidade & 2 & 2,0 \\
Mercado trabalho & 3 & 3,1 \\
Outro & 6 & 6,1 \\
Total & $\mathbf{9 8}$ & $\mathbf{1 0 0}$ \\
\hline
\end{tabular}

Tabela 3. Distribuição absoluta e percentual da principal finalidade e o motivo da escolha do curso de odontologia.

\begin{tabular}{lcc}
\hline & $\mathbf{n}$ & $\mathbf{\%}$ \\
\hline Finalidade da Odontologia & 63 & \\
Prevenção e manutenção & 19 & 64,3 \\
Prevenção e tratamento & 3 & 19,4 \\
Servir comunidade & 1 & 3,1 \\
Tratamento de doenças & 5 & 1,0 \\
Promover higiene e estética & 7 & 5,1 \\
Outro & $\mathbf{9 8}$ & 7,1 \\
Total & & $\mathbf{1 0 0}$ \\
& & \\
Porque você escolheu odontologia & 44 & 44,9 \\
Vocação & 37 & 37,8 \\
Profissão compensadora & 6 & 6,1 \\
Profissão liberal & 6 & 6,1 \\
Existência faculdade local & 5 & 5,1 \\
Não passei em outra & $\mathbf{9 8}$ & $\mathbf{1 0 0}$ \\
Total &
\end{tabular}

Quanto às dificuldades que possam encontrar no exercício profissional, quase a metade relatou a saturação do mercado de trabalho, razão pela qual $81,6 \%$ pretendem trabalhar simultaneamente em consultório particular e serviço público. Um estudo realizado em 2003 relata que o perfil predominante do profissional é aquele que trabalha no consultório particular próprio ou por porcentagem, sendo que
26,5\% atendem em algum Convênio ou Cooperativa e 12,2\% trabalham na rede pública $^{18}$. Por outro lado, outros estudos afirmam que os entrevistados após o termino do curso pretendiam trabalhar em consultório individual $^{3,13}$. Um estudo realizado no Ceará relatou que a maioria dos entrevistados pretendia trabalhar na rede pública ${ }^{14}$. Nota-se que ao longo dos anos, devido à saturação do mercado de 
trabalho, uma alternativa de estabilidade tem sido o serviço público.

\section{CONCLUSÃO}

Conclui-se que existe predominância de mulheres e os principais motivos que levaram os participantes a escolher a Odontologia como profissão foram a afinidade, o fato de ser uma profissão da área de saúde, influência da família, de amigos ou do cirurgião-dentista e o desejo de ajudar o ser humano.

A vocação foi a maior explicação para a procura do curso, e a necessidade de continuar estudando é preocupação constante. A maioria dos participantes acredita que o mercado de trabalho está saturado, mas almeja trabalhar seja em consultório individual, particular ou no serviço público.

\section{ABSTRACT \\ Profile of undergraduate students in Dentistry: professional motivations and expectations}

The purpose of this study was to register the profile of Dentistry undergraduate students regarding motivations and expectations. Participated in this research, 98 students of the Undergraduate Course in Dentistry at Faculdades Integradas de Patos, who fill out a questionnaire with objective and subjective questions. The results show that $68.4 \%$ were female, most from the state of Paraíba (67.3\%). Thereabouts $80 \%$ did entrance exams at another institution and $40.8 \%$ made two to three selective processes. The family income was predominant 3 to 6 minimum wages $(40.8 \%)$. The expectation of participants was training geared to work $(70.4 \%)$, the main reason for choosing was personal and professional achievement $(69.4 \%)$ and dentistry was one among other careers that could satisfy them (67.3\%). The results reveal that a good part of the interviewees are motivated in the professional and personal area and their expectations are the best geared towards the academic formation and the work. There is a predominance of women, most students believe that the job market is saturated, but they aim to work in individual, private, public or covenants. The vocation was the greatest explanation for the demand of the course, and the need to continue studying is a constant concern.

Descriptors: Dentistry Students. Training of Human Resources. Job Market.

\section{REFERÊNCIAS}

1. Matos MS, Tenório RM. Expectativas de estudantes de Odontologia sobre o campo de trabalho odontológico e o exercício profissional. Rev Bras Pesq Saúde. 2011; 13(4):10-21.

2. Ferreira NP, Ferreira AP, Freire MCM. Mercado de trabalho na Odontologia: contextualização e perspectivas. Rev Odontol UNESP. 2013;42(4):304-09.

3. Leite DFBM, Trigueiro M, Martins IMCLB, Lima Neto TJ, Santos MQ. Perfil socioeconômico de 253 graduandos de Odontologia de uma instituição privada em João Pessoa-PB em 2011. J Health Sci Inst. 2012;30(2):117-19.

4. Carvalho ACP. Planejamento do curso de graduação de Odontologia. Rev ABENO. 2004;4(1):7-13.

5. Machado DC, Souto DM, Freitas CHSM, Forte FDS. Odontologia como escolha: perfil de graduandos e perspectiva para o futuro profissional. Rev ABENO. 2010; 10(2):27-34.

6. Oliveira DL, Souza ES, Batista FJN, Alves JV, Yarid SD. Perfil do aluno de Odontologia da Universidade Estadual do Sudoeste da Bahia - UESB. Rev Saúde Com. 2013; 9(3):169-78.

7. Cardoso MB. As motivações acadêmicas e as expectativas profissionais dos alunos de odontologia da UFSC [monografia]. Florianópolis: Universidade Federal de Santa Catarina - UFSC; 2015.

8. Guimarães PDF, Medeiros MGA, Costa SM, Coelho MAQ, Andréa Maria Martins AMEBL, Pinto MQC. Perfil sociodemográfico dos estudantes de Odontologia: resultados parciais. In: Anais $9^{\circ}$ FEPEG, Montes Carlos. Minas Gerais: Campus Universitário Professor Darcy Ribeiro; 
2015.

9. Mialhe FL, Furuse R, Gonçalo CS. Perfil profissional de uma amostra de egressos da Faculdade de Odontologia de Piracicaba. Rev Odontol UFES. 2008;10(2):31-6.

10. Cavalcanti AL, Lima WG, Marques JLS, Alves HFC, Granville-Garcia AF. Motivos de ingresso e de evasão dos acadêmicos de Odontologia de uma instituição pública. Rev Odontol UNESP. 2010;39(2):95-9.

11. Brustolin J, Toassi RFC, Kunhnem M. Perfil do acadêmico de Odontologia da universidade do Planalto Catarinense. Rev ABENO. 2006;6(1):70-7.

12. Sousa JE, Maciel LKB, Zocratto KBF. O papel do ensino de graduação em Odontologia e o motivo da escolha da profissão: uma visão dos alunos concluintes. RFO UPF. 2013;18(3):277-83.

13. Souza LRF, Silva GD, Oliveira CAS, Zocratto KBF. Mercado de trabalho: perspectivas dos alunos do curso de Odontologia de uma faculdade particular d.e Belo Horizonte. Odontol Clín-Cient 2015; 14(3):707-12.

14. Araújo GS, Vasconcellos AA, Gomes ALF, Resende ISL, Lira RQN, Santiago SL. Estudo do perfil dos alunos recém-ingressos no curso de Odontologia da Universidade Federal do Ceará. In: Anais da $61^{\mathrm{a}}$ Reunião Anual da SBPC, Manaus. Amazonas: Universidade Federal do Amazonas; 2009.
15. Sundefeld MLMM, Perri SHV, Borghi WMMC, Rodrigues MAB. Escolhendo a profissão: opinião de alunos de Odontologia e Medicina Veterinária. Omnia Saúde. 2011; 8(1):36-49.

16. Marques MD, Souza CA, Pazos CTC, Amaral DS, Vieira EG, Campos WCS, et al. Expectativas dos estudantes de Odontologia quanto ao futuro profissional. Rev ABENO. 2015;15(3):60-8.

17. Rezende FP, Nakanishi FC, Machado ACP, Quirino MRS, Anbinder AL. Perfil, motivações e expectativas dos graduandos e graduados em Odontologia. Rev Odontol Univ Cid São Paulo. 2007;19(2):165-72.

18. Bastos JRM, Aquilante AG, de Almeida BS, Lauris JRP, Bijella VT. Análise do perfil profissional de cirurgiões-dentistas graduados na Faculdade de Odontologia de Bauru-USP entre os anos de 1996 e 2000. J Appl Oral Sci. 2003;11(4):283-9.

Correspondência para:

Gélica Lima Granja

e-mail: gelicagranja@hotmail.com

Avenida Manoel dos Arroz, $\mathrm{n}^{\circ}$ 85, bairro

56306-385 Vila Mocó, Petrolina/PE 